\title{
Tuning the charge density wave quantum critical point and the appearance of superconductivity in $\mathrm{TiSe}_{2}$
}

\author{
Sangyun Lee $\odot,{ }^{1,2,3,{ }^{*}}$ Tae Beom Park, ${ }^{1}$ Jihyun Kim, ${ }^{1}$ Soon-Gil Jung, ${ }^{1}$ Won Kyung Seong, ${ }^{1, \dagger}$ \\ Namjung Hur, ${ }^{4}$ Yongkang Luo, ${ }^{3, *}$ Duk Y. Kim, ${ }^{2}$ and Tuson Park ${ }^{1, \S}$ \\ ${ }^{1}$ Center for Quantum Materials and Superconductivity (CQMS) and Department of Physics, \\ Sungkyunkwan University, Suwon 16419, Republic of Korea \\ ${ }^{2}$ Center for Integrated Nanostructure Physics (CINAP), Institute for Basic Science (IBS), Suwon 16419, Republic of Korea \\ ${ }^{3}$ Los Alamos National Laboratory, Los Alamos, New Mexico 87545, USA \\ ${ }^{4}$ Department of Physics, Inha University, Incheon 22212, Republic of Korea
}

(Received 23 January 2021; revised 22 June 2021; accepted 24 June 2021; published 28 July 2021)

\begin{abstract}
The transition metal dichalcogenide $\mathrm{TiSe}_{2}$ is an ideal correlated system for studying the interplay between superconductivity (SC) and a charge density wave (CDW) because both symmetry-breaking phases can be easily controlled by either $\mathrm{Cu}$ intercalation or physical pressure. SC appears in proximity to a CDW quantum critical point (QCP) induced by both $\mathrm{Cu}$ intercalation and applied pressure, raising the possibility of CDW-driven SC. Here, we report tuning the CDW QCP by simultaneously controlling $\mathrm{Cu}$ intercalation and external pressure and the appearance of a SC dome centered on the tunable QCP. When subjected to pressure, CDW ordering of $\mathrm{Cu}$ intercalated $\mathrm{Cu}_{0.025} \mathrm{TiSe}_{2}$ is completely suppressed at $2.3 \mathrm{GPa}$, where the residual resistivity and the resistivitytemperature exponent decrease sharply, indicating the presence of the CDW QCP. The upper critical field of $\mathrm{Cu}_{0.025} \mathrm{TiSe}_{2}$ is $3.51 \mathrm{kOe}, 16$ times larger than that of pristine $\mathrm{TiSe}_{2}$, and its temperature dependence is linear, indicating that $\mathrm{SC}$ of $\mathrm{TiSe}_{2}$ is switched from the two-dimensional- to anisotropic three-dimensional-like by $\mathrm{Cu}$ intercalation. These discoveries show that the simultaneous application of $\mathrm{Cu}$ intercalation and pressure move the CDW QCP and that the highest SC transition temperature is pinned to the QCP, suggesting that the SC in $\mathrm{TiSe}_{2}$ is strongly correlated with CDW quantum criticality.
\end{abstract}

DOI: 10.1103/PhysRevResearch.3.033097

\section{INTRODUCTION}

A charge density wave (CDW) is a quantum state in which electrons at the Fermi energy organize themselves into a collective mode with a gap in the single-particle excitation spectrum [1]. Because the Fermi surface of low-dimensional materials is particularly susceptible to an instability induced by charge and atomic lattice modulation associated with a CDW, these materials are prone to host a CDW. Observation of CDW order in proximity to the superconducting (SC) phase of high- $T_{c}$ cuprates and other low-dimensional systems raises the fundamental question of whether the two symmetrybreaking phases compete, coexist, or cooperate [2-6]. Most

\footnotetext{
*hansan29@skku.edu

†Present address: Center for Multidimensional Carbon Materials (CMCM), Institute for Basic Science (IBS), Ulsan 44919, Republic of Korea.

*Present address: Wuhan National High Magnetic Field Center, Huazhong University of Science and Technology, Wuhan 430074, Hubei, China.

${ }^{\S}$ Corresponding author: tp8701@skku.edu

Published by the American Physical Society under the terms of the Creative Commons Attribution 4.0 International license. Further distribution of this work must maintain attribution to the author(s) and the published article's title, journal citation, and DOI.
}

studies have focused on tuning the fermiology by applying a single nonthermal control parameter, establishing that static CDW and SC orders compete for parts of the Fermi surface. The interplay between SC in low-dimensional correlated systems and quantum fluctuations associated with the CDW quantum critical point (QCP), however, is still being debated.

The two-dimensional (2D) transition metal dichalcogenide (TMD) $\mathrm{TiSe}_{2}$, in which Se-Ti-Se layers are separated by a van der Waals gap [7], has been extensively studied to probe the interplay between $\mathrm{SC}$ and $\mathrm{CDW}$ via various external parameters such as doping [8-11], intercalation [12-17], pressure [18-21], and layer thickness [22-25]. The maximal SC transition temperature $\left(T_{c, \max }\right)$ emerges at a point where the CDW transition temperature is suppressed to zero by external pressure and/or $\mathrm{Cu}$ intercalation $[12,18]$. An x-ray scattering study of $\mathrm{TiSe}_{2}$ under high pressure reveals, however, that the CDW state in $\mathrm{TiSe}_{2}$ is separated into a commensurate CDW (CCDW) and an incommensurate CDW (ICDW) under pressure. The CCDW state disappears at the optimal pressure, showing $T_{c, \max }(\sim 3.0 \mathrm{GPa})$ and indicating that the CCDW and $\mathrm{SC}$ orders of $\mathrm{TiSe}_{2}$ are closely related [13,14,16,17,26,27]. In contrast, the ICDW state is not suppressed even at a pressure of 5.1 GPa, suggesting that the SC and ICDW orders proceed to develop separately $[10,19]$.

The effects of external pressure and $\mathrm{Cu}$ intercalation in $\mathrm{TiSe}_{2}$ are similar in that both suppress the CDW order and induce SC. By contrast, the change in other physical properties 
depends on the control parameter $[13,18]$. For example, the sign of the Hall coefficient $\left(R_{\mathrm{H}}\right)$ is positive for pristine $\mathrm{TiSe}_{2}$, whereas it is negative for $\mathrm{Cu}_{x} \mathrm{TiSe}_{2}$ because a small amount of $\mathrm{Cu}$ intercalation $(x=0.015)$ changes the major charge carrier type from hole to electron [13]. The upper critical field $\left(H_{c 2}\right)$ for pressure-induced $\mathrm{SC}$ of $\mathrm{TiSe}_{2}$ is $0.23 \mathrm{kOe}$ $\left(T_{c}=0.7 \mathrm{~K}\right.$ at $\left.3.5 \mathrm{GPa}\right)$, whereas that for the $\mathrm{Cu}$-intercalated $\mathrm{Cu}_{0.07} \mathrm{TiSe}_{2}$ is $12.6 \mathrm{kOe}\left(T_{c}=3.9 \mathrm{~K}\right.$ at ambient pressure), an enhancement in $H_{c 2}$ of 55 times [17,18]. Maschek et al. [26] considered two different pairing mechanisms in the $\mathrm{Cu}$ intercalation $(x)$-pressure-temperature $(x-P-T)$ phase diagram of $\mathrm{TiSe}_{2}$ : enhanced electron-phonon coupling provided by phonon-mode softening as the CDW QCP is approached with $\mathrm{Cu}$-intercalation at atmospheric pressure and by pressureenhanced hybridization of a hybrid phonon-exciton mode that softens in proximity to the pressure-induced CDW QCP in pure $\mathrm{TiSe}_{2}$. Therefore, simultaneously controlling both pressure and $\mathrm{Cu}$ intercalation is crucial to deepening our understanding of the origin of $\mathrm{SC}$ in the $\mathrm{CDW} \mathrm{TiSe}_{2}$ system and exploring the possibility of CDW-driven SC.

Here, we report electrical resistivity measurements on $\mathrm{Cu}-$ intercalated $\operatorname{TiSe}_{2}(x=0.025)$ under pressures as high as 3.1 $\mathrm{GPa}$. Like the pressure effects on pristine $\mathrm{TiSe}_{2}$, the SC state of $\mathrm{Cu}_{0.025} \mathrm{TiSe}_{2}$ is induced by the application of pressure, while the CDW transition temperature $\left(T_{\mathrm{CDW}}\right)$ is suppressed. Our comprehensive $x-P-T$ phase diagram shows that the CDW state is suppressed at a critical pressure where $T_{c}$ reaches its maximum. The upper critical field $\left(H_{c 2}\right)$ of $\mathrm{Cu}_{0.025} \mathrm{TiSe}_{2}$ at $3.1 \mathrm{GPa}$ is $3.51 \mathrm{kOe}$, and its temperature dependence is quasilinear, which is significantly different from that of pristine $\mathrm{TiSe}_{2}$ under pressure $(3.5 \mathrm{GPa})[18,19]$, but is similar to $\mathrm{Cu}$-intercalated $\mathrm{Cu}_{0.07} \mathrm{TiSe}_{2}$ at ambient pressure $[12,17]$. These results imply that $\mathrm{Cu}$ intercalation between the $\mathrm{Se}-\mathrm{Ti}-\mathrm{Se}$ layers separated by a van der Waals gap in $\mathrm{Cu}_{x} \mathrm{TiSe}_{2}$ induces a dimensional crossover from 2D- to three-dimensional (3D)like SC properties in $\mathrm{TiSe}_{2}$. Our results that the maximal $T_{c}$ is pinned to the $\mathrm{CDW}$ QCP without regarding $\mathrm{Cu}$ intercalation, external pressure, or simultaneous application of both control parameters reflect that there is a strong correlation between CDW QCP and the SC of $\mathrm{TiSe}_{2}$.

\section{EXPERIMENT}

$\mathrm{Cu}$-intercalated $\mathrm{TiSe}_{2}$ single crystals, $\mathrm{Cu}_{x} \mathrm{TiSe}_{2}$ with $x=$ 0.025 , were synthesized by the vapor transport method $[12,28]$. The crystal structure and chemical composition ratio of the fabricated $\mathrm{Cu}_{x} \mathrm{TiSe}_{2}$ single crystals were determined using a powder x-ray diffractometer (Rigaku miniflex-600) and energy-dispersive $\mathrm{x}$-ray spectrometer (EDX), respectively. EDX scans for the overall area of the sample showed $x=$ $0.025 \pm 0.003$ on average, while $x$ ranges from 0.027 to 0.021 at some specific sites such as craters. Both $a$ - and $c$-axis lattice parameters obtained from powder x-ray diffraction (PXRD) measurements are also consistent with previous results [12], indicating that crystals of $\mathrm{Cu}_{0.025} \mathrm{TiSe}_{2}$ are reproducible and homogeneously synthesized. The standard four-point contact technique was used to measure the electrical resistivity $(\rho)$ of the $\mathrm{Cu}_{x} \mathrm{TiSe}_{2}$ samples that were prepared in bar shapes, and we had screened many crystals to select the best quality sample for the pressure experiment. Quasihydrostatic pressure
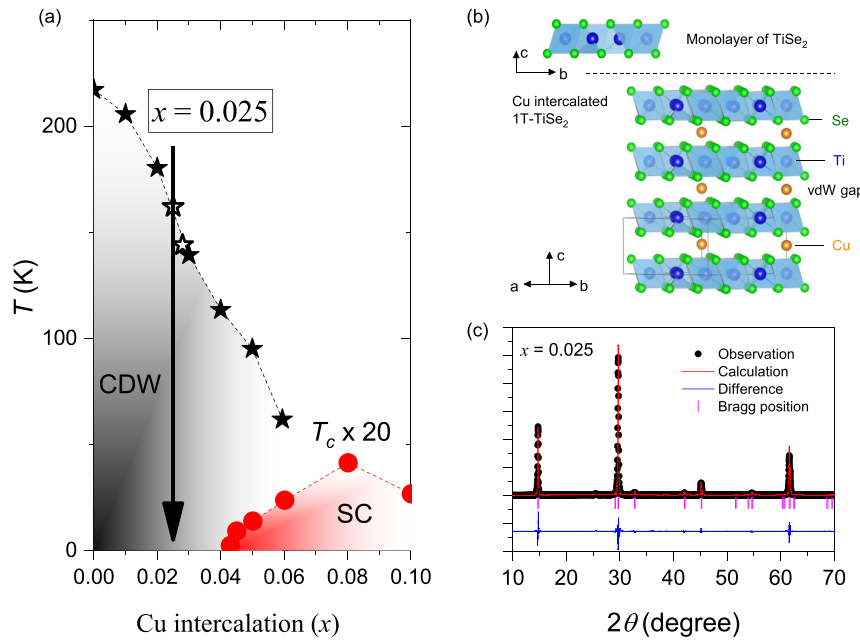

FIG. 1. (a) Schematic phase diagram of $\mathrm{Cu}_{x} \mathrm{TiSe}_{2}$ as a function of the degree of $\mathrm{Cu}$ intercalation [12]. The concentration $x=0.025$ is marked with an arrow, and open symbols represent current experiments. (b) Crystal structure of $\mathrm{Cu}_{x} \mathrm{TiSe}_{2}$. The Ti atom is located at the center of an octahedral structure formed by six Se atoms. These structures form $\mathrm{Se}-\mathrm{Ti}-\mathrm{Se}$ layers (the $\mathrm{Ti}$ and $\mathrm{Se}$ atoms are blue and green, respectively). The $\mathrm{Cu}$ atoms (orange) are intercalated in the van der Waals gap between the Se-Ti-Se layers. (c) Powder x-ray diffraction pattern (PXRD) of the $x=0.025$ sample is plotted as a function of the angle and compared with the calculated pattern obtained via Rietveld refinement.

environments were achieved using a silicone fluid and a glycerol-water mixture (60/40) as a pressure transmitting medium for pressures up to 2.5 and $3.5 \mathrm{GPa}$ in a cylindrical clamp-type and an indenter-type cell, respectively [29,30]. The pressure inside the cell was determined by measuring the suppression of the SC transition temperature of $\mathrm{Sn}$ and $\mathrm{Pb}$ [31], and they showed a sharp SC transition at all pressures, reflecting that the pressure was applied smoothly. A custom-built helium-pumping cryostat was used for resistivity measurements from 1.4 to $300 \mathrm{~K}$. The SC properties down to $0.3 \mathrm{~K}$ were studied by applying an external magnetic field using the Oxford Heliox VL system.

\section{RESULTS AND DISCUSSION}

Figure 1(a) shows the temperature vs Cu intercalation $(T-x)$ phase diagram, with the location of $x=0.025$ marked by the arrow. Energy-dispersive x-ray spectroscopy (EDS) measurements were used to determine the actual ratio of intercalated $\mathrm{Cu}$ atoms in $\mathrm{TiSe}_{2}$ crystals. Figure 1(c) shows the $\theta-2 \theta$ scans of the PXRD patterns of $\mathrm{Cu}_{x} \mathrm{TiSe}_{2}$ with $x=0.025$. The $a$ and $c$-axis lattice parameters that were calculated using FULLPROF software are 6.017 and $3.54 \AA$, which are consistent with those in a previous study [12]. Both $a$ and $c$ increase smoothly with the $\mathrm{Cu}$ content, indicating that $\mathrm{Cu}$ atoms are successfully intercalated in the van der Waals gap, separating the Se-Ti-Se layers with ionic bonding [12], as illustrated in Fig. 1(b).

Figure 2(a) shows the temperature dependence of the electrical resistivity $(\rho)$ of $\mathrm{Cu}_{0.025} \mathrm{TiSe}_{2}$ under pressure. Values of $\rho$ at room temperature $\rho(300 \mathrm{~K})$ are reduced by the 
(a)

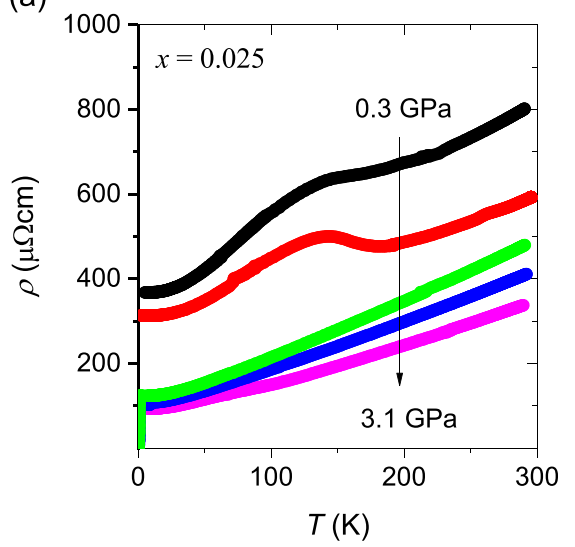

(b)

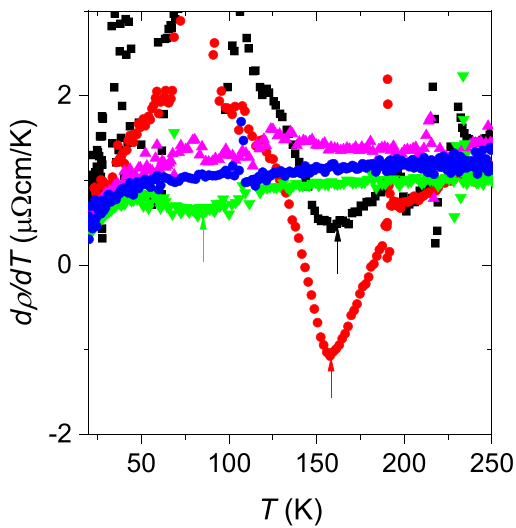

(c)

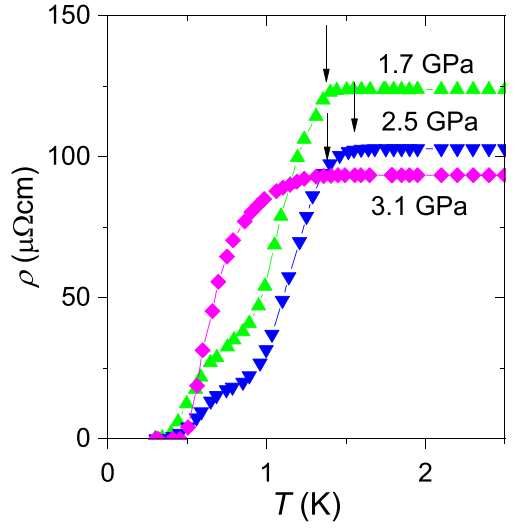

(d)

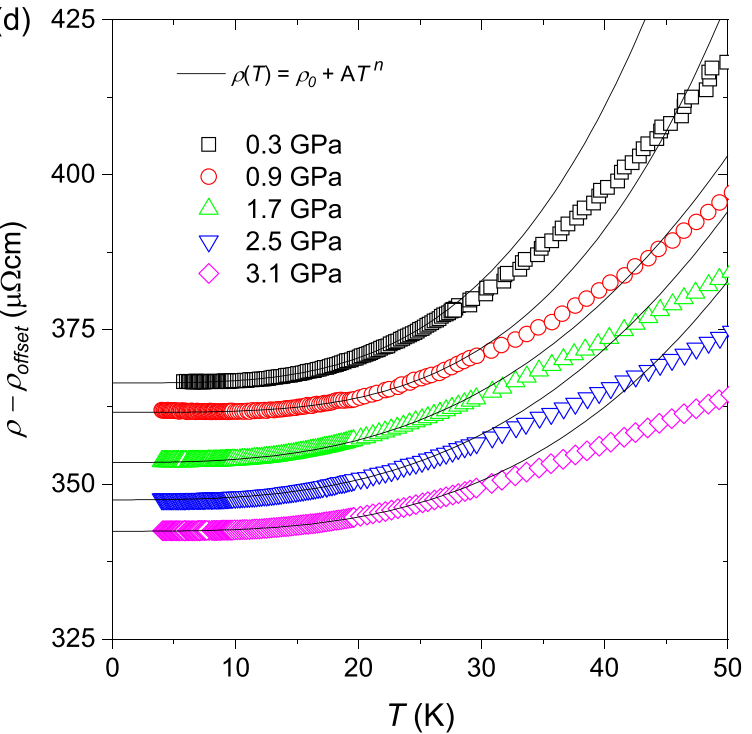

(e)

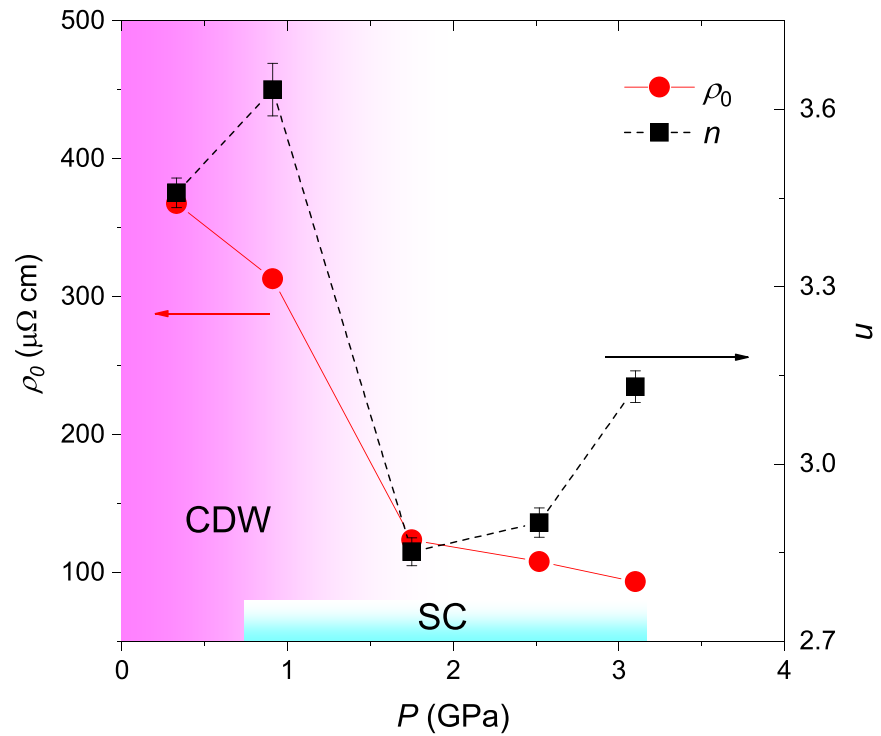

FIG. 2. Electrical resistivity of $\mathrm{Cu}_{0.025} \mathrm{TiSe}_{2}$. The electrical resistivity $(\rho)$ and its first derivative $(d \rho / d T)$ are plotted for pressures of 0.3 , $0.9,1.7,2.5$, and $3.1 \mathrm{GPa}$ in (a) and (b), respectively. The arrows in (b) mark the minimum $d \rho / d T$, which are assigned as $T_{\mathrm{CDW}}$. (c) Magnified view of $\rho$ near $T_{c}$ plotted for 1.7, 2.5, and 3.1 GPa, where the down arrows mark the onset of the superconducting phase transition. (d) Analysis of the low-temperature resistivity by using the power-law form of $\rho(T)=\rho_{0}+A T^{n}$, where the best result of the least squares fitting is plotted as a black curve. $\rho(T)$ is rigidly shifted for clarity with an offset offset ( $\left.\rho_{\text {offset }}\right)$ against values at ambient pressure ( 1 bar), where it is 48,230 , 245, and 250 for $0.9,1.7,2.5$, and $3.1 \mathrm{GPa}$, respectively. (e) Overlay of the residual resistivity $\left(\rho_{0}\right)$ and the temperature exponent $n$ in the $T$ vs $P$ phase diagram. A sharp reduction of $\rho_{0}$ and $n$ is observed in the vicinity of $P_{c}$, where $T_{c}$ is maximum.

application of external pressure because of the increased overlap between adjacent orbitals. This large reduction of $\rho(300$ $\mathrm{K})$ under pressure has been widely observed in TMDs, which consist of stacked layers with a van der Waals gap $[32,33]$. Figure 2(b) displays the first derivative of the resistivity $(d \rho / d T)$ as a function of the temperature, where a dip feature corresponds to $T_{\mathrm{CDW}}$, as indicated by the arrows. Because any features associated with an ICDW in $\mathrm{TiSe}_{2}$ are difficult to observe by electrical resistivity measurements [19], we assume that the dip in $d \rho / d T$ arises from the CCDW phase transition. When subjected to pressure, the $T_{\mathrm{CDW}}$ of $\mathrm{Cu}_{0.025} \mathrm{TiSe}_{2}$ decreases and disappears at the critical pressure $\left(P_{c}\right)$ near $2.3 \mathrm{GPa}$, where $T_{c}$ is the maximum $\left(T_{c, \text { max }}\right)$.

Figure 2(c) shows a magnified view of the electrical resistivity of $\mathrm{Cu}_{0.025} \mathrm{TiSe}_{2}$ near the SC phase transition, at which the onset of $T_{c}$ is $\sim 1.4 \mathrm{~K}$ at $1.7 \mathrm{GPa}$. We note that the SC phase transition occurs in broader temperature ranges at 1.7 and $2.5 \mathrm{GPa}$ compared with $3.1 \mathrm{GPa}$. Similar broadening in the SC phase transition is also observed in materials in close proximity to a QCP, where SC coexists with other ordered states such as CDW and antiferromagnetic (AFM) phases $[12,32,34]$. To make a better understanding, however, we need further analysis via such spectroscopic studies as muon spin spectroscopy $(\mu \mathrm{SR})$ or nuclear magnetic resonance (NMR) measurements. The temperature dependence of the resistivity in the normal state $\left(T>T_{c}\right)$ is plotted with an offset for clarity in Fig. 2(d) and was analyzed using the power-law form of $\rho(T)=\rho_{0}+A T^{n}$. As shown in Fig. 2(e), the residual resistivity $\rho_{0}$ decreases with increasing pressure for $P<P_{c}$, whereas it is almost saturated above $P_{c}$, indicating that the rapid decrease in $\rho_{0}$ is closely related to the destruction of the CDW gap. The resistivity temperature exponent $n$, which is displayed by the square symbols on the right ordinate of Fig. 2(e), is $>2$, which is expected from Landau-Fermi liquids in normal metals. An anomalously large exponent $n$ was also observed in pristine $\mathrm{TiSe}_{2}$ under pressure, which was ascribed 

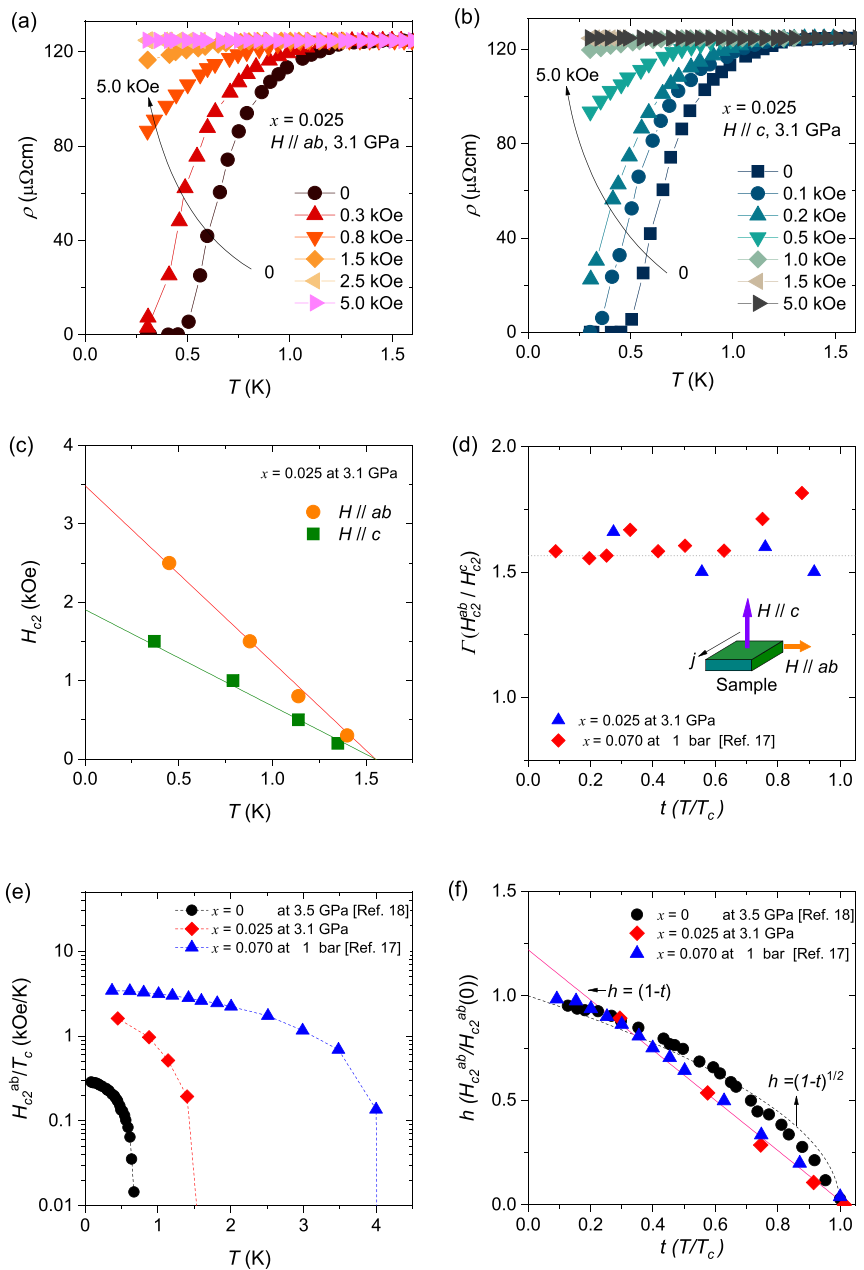

FIG. 3. Temperature-dependent upper critical field $\left(H_{c 2}\right)$ of $\mathrm{Cu}_{0.025} \mathrm{TiSe}_{2}$. Electrical resistivity for the magnetic field applied within $(H \| a b)$ and perpendicular to the plane $(H \| c)$ plotted as a function of temperature in (a) and (b), respectively. (c) Temperature dependences of the upper critical field plotted for $H \| a b$ (circles) and $H \| c$ (squares), obtained from the onset of the superconductivity (SC) transition temperature $T_{c \text {,onset }}$. (d) The anisotropy ratio of the upper critical field $\left(\Gamma=H_{c 2}^{a b} / H_{c 2}^{c}\right)$. Notice that $\Gamma$ is almost independent of temperature. (e) Upper critical field divided by $T_{c}$. The data for $x=0$ and 0.07 are from Refs. [17,18], respectively. (f) Reduced upper critical field $(h)$ plotted as a function of the reduced temperature $(t)$ and fitted with the linear and square root forms of $h=\alpha_{i}(1-t)$ and $h=(1-t)^{1 / 2}$, respectively, where the best results are sequentially plotted using solid and dotted lines.

to a phonon-assisted interband scattering mechanism [18]. With increasing pressure, $n$ increases slightly from 3.5 to 3.6 at $0.9 \mathrm{GPa}$, is sharply reduced to a minimum $(n \approx 2.85)$ near $2.3 \mathrm{GPa}\left(=P_{c}\right)$, and gradually increases with further application of pressure. We note that a similar pressure dependence of $n$ was observed for pristine $\mathrm{TiSe}_{2}$ and was explained in terms of the quantum fluctuations associated with the CCDW QCP [18].

The low-temperature resistivities of $\mathrm{Cu}_{0.025} \mathrm{TiSe}_{2}$ at 3.1 GPa are represented for magnetic fields applied parallel $(H \| a b)$ and perpendicular $(H \| c)$ to the $a b$ plane in Figs. 3(a) and 3(b), respectively. As shown in Fig. 3(c), the upper critical field $H_{c 2}$ obtained with the criterion of the onset of SC transition ( $=T_{c \text {,onset }}$ ) increases linearly with decreasing temperature for both field orientations and extrapolates to 1.93 and $3.51 \mathrm{kOe}$ at zero-temperature limit for $H_{c 2}^{c}(0)$ and $H_{c 2}^{a b}(0)$, respectively. Although $T_{c}$ of the $\mathrm{Cu}$-intercalated material increases twice as fast as that of pristine $\mathrm{TiSe}_{2}$, its $H_{c 2}^{a b}(0)$ is one order of magnitude larger than that $(=0.22 \mathrm{kOe})$ of pristine $\mathrm{TiSe}_{2}$. The Ginzburg-Landau coherence lengths can be estimated as $\xi_{a b}(0)=414 \AA\left[H_{c 2}^{c}(0)=\phi_{0} / 2 \pi \xi_{a b}^{2}(0)\right]$ and $\xi_{c}=$ $226 \AA \quad\left[H_{c 2}^{a b}(0)=\phi_{0} / 2 \pi \xi_{a b}(0) \xi_{c}(0)\right]$, where $\phi_{0}(=2.07 \times$ $\left.10^{-15} \mathrm{Tm}^{2}\right)$ is the quantized magnetic flux [35]. Figure 3(d) shows that the $H_{c 2}$ anisotropy $\left(\Gamma=H_{c 2}^{a b} / H_{c 2}^{c}\right)$ of the compound with $x=0.025$ is $\sim 1.6$ and is almost independent of the temperature, which is like the $\Gamma(\sim 1.7)$ of $\mathrm{Cu}_{0.07} \mathrm{TiSe}_{2}$ at ambient pressure [17].

Figure 3(e) shows $H_{c 2}^{a b}(T)$ divided by $T_{c}, H_{c 2}^{a b}(0) / T_{c}$, for pristine and $\mathrm{Cu}$-intercalated $\mathrm{TiSe}_{2}$ on a semilogarithmic scale. The upper critical field ratio of the $\mathrm{Cu}$-intercalated $\mathrm{TiSe}_{2}$ was $0.28,2.21$, and $3.42 \mathrm{kOe} / \mathrm{K}$ for the pristine, $x=0.025$, and $x=0.070$ materials, respectively. Figure 3(f) displays the reduced upper critical field $h=H_{c 2}^{a b}(T) / H_{c 2}{ }^{a b}(0)$ of the pristine (circles), $\mathrm{Cu}_{0.025} \mathrm{TiSe}_{2}$ (diamonds), and $\mathrm{Cu}_{0.07} \mathrm{TiSe}_{2}$ (triangles) as a function of the reduced temperature $t=T / T_{c \text {,onset }}$. Here, $h(t)$ of pristine $\mathrm{TiSe}_{2}$ at $3.5 \mathrm{GPa}$ is well expressed by the $2 \mathrm{D}$ behavior, that is, $h(t)=(1-t)^{1 / 2}$. In contrast, $h(t)$ of both $\mathrm{Cu}$-intercalated $\mathrm{TiSe}_{2}$ shows a $t$-linear behavior, indicating a crossover from 2D- to 3D-like SC behavior via $\mathrm{Cu}$ intercalation [36]. Similar effects have also been reported in the dependence of the $c$-axis resistivity $\left(\rho_{c}\right)$ on $\mathrm{Cu}$-intercalation level $[13,37]$. With increasing $\mathrm{Cu}$ intercalation, $\rho_{c}$ rapidly decreases from $40 \Omega \mathrm{cm}$ at $x=0$ to $17 \Omega \mathrm{cm}$ at $x=0.015$, indicating that only a small amount of $\mathrm{Cu}$ intercalation has a significant effect on interlayer coupling and, thereby, the dimensional crossover in SC properties.

Figure 4(a) compares the temperature-pressure phase diagram of pristine $\mathrm{TiSe}_{2}$ (open symbols) and $\mathrm{Cu}$-intercalated $\mathrm{Cu}_{0.025} \mathrm{TiSe}_{2}$ (solid symbols) systems. In both compounds, $T_{\mathrm{CDW}}$ is gradually suppressed under pressure and extrapolates to zero temperature at 3.0 and $2.3 \mathrm{GPa}$ for the pristine and $x=0.025$ compounds, respectively. Commensurate with the change in $T_{\mathrm{CDW}}$, the pressure-induced $\mathrm{SC}$ dome of $\mathrm{Cu}_{0.025} \mathrm{TiSe}_{2}$ shifts to the lower pressure side and shows a maximum at the projected CDW QCP of $2.3 \mathrm{GPa}$, indicating the strong correlation between the CDW and SC orders in the $\mathrm{TiSe}_{2}$ system. Because the minimum of the first derivative of the resistivity of $\mathrm{TiSe}_{2}$ corresponds to the CCDW transition temperature determined from the high-pressure $\mathrm{X}$-ray scattering experiment $[18,19]$, the CDW QCP in $\mathrm{Cu}_{0.025} \mathrm{TiSe}_{2}$ could be related to the suppression of the CCDW, supporting that CCDW is related to the SC order, whereas the ICDW behaves entirely independently. We note that these results are inconsistent with SC produced by the hybridization of exciton and phonon modes: the CDW QCP and $T_{c}$ maximal pressure was predicted to be different in the model [26], while our results show that they occur at the same point. To make a clearer understanding of SC mechanism, more experimental as well as theoretical work on SC gap is required at various intercalation levels.

Figure 4(b) shows an $x-P-T$ phase diagram for CDW $\mathrm{Cu}_{x} \mathrm{TiSe}_{2}$ superconductors $[12,14,17,18]$. In the $x$ - $T$ plane 


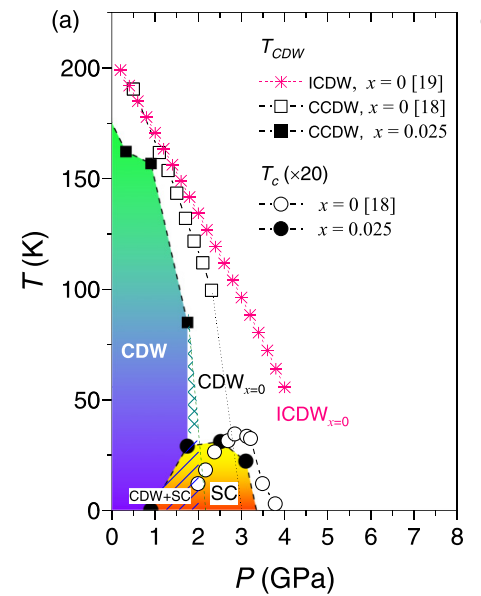

(b)

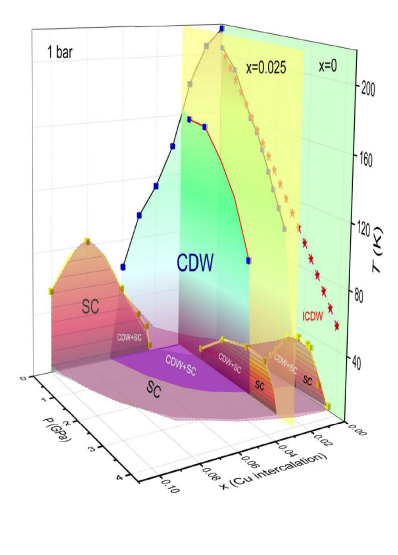

FIG. 4. (a) Temperature vs pressure phase diagram of $\mathrm{Cu}_{x} \mathrm{TiSe}_{2}$. The solid and open symbols represent $T_{\mathrm{CDW}}$ (squares) and $T_{c}$ (circles) for the $x=0.025$ and 0 compounds [18,19], respectively. The dotted line for $x=0.025$ is an extrapolated phase boundary that ends at $2.3 \mathrm{GPa}$, the projected charge density wave (CDW) quantum critical point (QCP). (b) $\mathrm{Cu}$ intercalation-pressure-temperature $(x-P-T)$ phase diagram representing three datasets: (i) at ambient pressure, $T_{\mathrm{CDW}}$ and $T_{c}$ in the $x-T$ plane, (ii) at $x=0, T_{\mathrm{CDW}}$ and $T_{c}$ in the $P-T$ plane, and (iii) at $x=0.025, T_{\mathrm{CDW}}$ and $T_{c}$ in the $P-T$ plane. The results from previous studies [12,14,17-19] are plotted together with those from this paper. The superconductivity (SC) transition temperature $\left(T_{c}\right)$ is multiplied by 20 for visibility.

at ambient pressure, the CDW ordering temperature is suppressed with $\mathrm{Cu}$ intercalation and is projected to $0 \mathrm{~K}$ at $x_{c}=0.07$, where the SC transition temperature reaches a maximum. The coincidence of the projected CDW QCP and maximum $T_{c}$ suggests the possibility of CDW-driven SC in $\mathrm{TiSe}_{2}$. Like $\mathrm{Cu}$ intercalation, the application of external pressure to pristine $\mathrm{TiSe}_{2}$, as displayed in the $P-T$ plane at $x=0$, reveals that the CDW order is completely suppressed at the critical pressure $P_{c}$, where the induced SC transition temperature is maximal. Although the separate application of
$\mathrm{Cu}$ intercalation and external pressure reveals a close connection between the CDW QCP and SC, it has been argued to be coincidental in the sense that each tuning parameter may produce an SC phase of which the mechanism is different [26]. By contrast, the $\mathrm{Cu}_{0.025} \mathrm{TiSe}_{2}$ was adjusted using both control parameters simultaneously and succeeded in moving the CDW QCP from 3.0 to 2.3 GPa. Together with the moved QCP, the SC dome is shifted to lower pressure, and the $T_{c}$ maximum is pinned to the shifted QCP, evidencing that critical fluctuations associated with the CCDW QCP produce the necessary attractive interaction to form SC electron pairs.

In conclusion, we report that the CDW QCP of $\mathrm{TiSe}_{2}$ can be adjusted via the simultaneous application of $\mathrm{Cu}$ intercalation and physical pressure. The $\mathrm{CDW}$ transition temperature of $\mathrm{Cu}$-intercalated $\mathrm{Cu}_{x} \mathrm{TiSe}_{2}(x=0.025)$ decreases with increasing pressure and is terminated near $2.3 \mathrm{GPa}$, the optimal pressure $\left(P_{c}\right)$ at which $T_{c}$ is the maximum. The upper critical field of the $\mathrm{Cu}$-intercalated compound is $3.51 \mathrm{kOe}, 16$ times larger than that of the pristine compound, and its temperature dependence changes from a square root form for the pristine compound to a linear one for $x=0.025$, indicating a crossover from 2D- to 3D-like SC behavior via $\mathrm{Cu}$ intercalation. Control of SC dome via the tunable CDW QCP in $\mathrm{Cu}_{x} \mathrm{TiSe}_{2}$ is like the $f$-electron-based AFM superconductor $\mathrm{CeRhIn}$, where the SC dome is pinned to the moved AFM QCP via Sn doping [38]. In addition, our finding that the SC dome of $\mathrm{TiSe}_{2}$ is shifted along with CDW QCP suggests the possibility of a class of strongly correlated SC systems where SC and CDW are intertwined.

\section{ACKNOWLEDGMENTS}

We acknowledge a fruitful discussion with J. D. Thompson. The work at Sungkyunkwan University was supported by a NRF grant funded by the Ministry of Science, ICT and Future Planning (No. 2012R1A3A2048816). The work at Los Alamos was performed under the auspices of the U.S. Department of Energy, Office of Science, Basic Energy Sciences, Materials Sciences and Engineering Division.
[1] G. Grüner, The dynamics of charge-density waves, Rev. Mod. Phys. 60, 1129 (1988).

[2] J. Chang, E. Blackburn, A. T. Holmes, N. B. Christensen, J. Larsen, J. Mesot, R. Liang, D. A. Bonn, W. N. Hardy, A. Watenphul, M. v. Zimmermann, E. M. Forgan, and S. M. Hayden, Direct observation of competition between superconductivity and charge density wave order in $\mathrm{YBa}_{2} \mathrm{Cu}_{3} \mathrm{O}_{6.67}$, Nat. Phys. 8, 871 (2012).

[3] A. Chikina, A. Fedorov, D. Bhoi, V. Voroshnin, E. Haubold, Y. Kushnirenko, K. H. Kim, and S. Borisenko, Turning chargedensity waves into Cooper pairs, npj Quantum Mater. 5, 22 (2020).

[4] Y. Feng, J. Wang, R. Jaramillo, J. van Wezel, S. Haravifard, G. Srajer, Y. Liu, Z.-A. Xu, P. B. Littlewood, and T. F. Rosenbaum, Order parameter fluctuations at a buried quantum critical point, Proc. Natl Acad. Sci. USA 109, 7224 (2012).

[5] K. Cho, M. Kończykowski, S. Teknowijoyo, M. A. Tanatar, J. Guss, P. B. Gartin, J. M. Wilde, A. Kreyssig, R. J. McQueeney,
A. I. Goldman, V. Mishra, P. J. Hirschfeld, and R. Prozorov, Using controlled disorder to probe the interplay between charge order and superconductivity in $\mathrm{NbSe}_{2}$, Nat. Comms. 9, 2796 (2018).

[6] D. Bhoi, S. Khim, W. Nam, B. S. Lee, C. Kim, B.-G. Jeon, B. H. Min, S. Park, and K. H. Kim, Interplay of charge density wave and multiband superconductivity in $2 \mathrm{H}-\mathrm{Pd}_{x} \mathrm{TaSe}_{2}$, Sci. Rep. 6, 24068 (2016).

[7] F. J. Di Salvo, D. E. Moncton, and J. V. Wazczak, Electronic properties and superlattice formation in the semimetal $\mathrm{TiSe}_{2}$, Phys. Rev. B 14, 4321 (1976).

[8] M. M. May, C. Brabetz, C. Janowitz, and R. Manzke, ChargeDensity-Wave Phase of $1 T$-TiSe 2 : The Influence of Conduction Band Population, Phys. Rev. Lett. 107, 176405 (2011).

[9] H. Luo, J. W. Krizan, E. M. Seibel, W. Xie, G. S. Sahasrabudhe, S. L. Bergman, B. F. Phelan, J. Tao, Z. Wang, J. Zhang, and R. J. Cava, Cr-doped $\mathrm{TiSe}_{2}$ - a layered dichalcogenide spin glass, Chem. Mater. 27, 6810 (2015). 
[10] H. Luo, W. Xie, J. Tao, H. Inoue, A. Gyenis, J. W. Krizan, A. Yazdani, Y. Zhu, and R. J. Cava, Polytypism, polymorphism, and superconductivity in $\mathrm{TaSe}_{2-x} \mathrm{Te}_{x}$, Proc. Natl. Acad. Sci. USA 112, E1174 (2015).

[11] N. Giang, Q. Xu, Y. S. Hor, A. J. Williams, S. E. Dutton, H. W. Zandbergen, and J. Cava, Superconductivity at $2.3 \mathrm{~K}$ in the misfit compound $(\mathrm{PbSe})_{1.16}\left(\mathrm{TiSe}_{2}\right)_{2}$, Phys. Rev. B 82, 024503 (2010).

[12] E. Morosan, H. W. Zandbergen, B. S. Dennis, J. W. G. Bos, Y. Onose, T. Klimczuk, A. P. Ramirez, N. P. Ong, and R. J. Cava, Superconductivity in $\mathrm{Cu}_{x} \mathrm{TiSe}_{2}$, Nat. Phys. 2, 544 (2006).

[13] G. Wu, H. X. Yang, L. Zhao, X. G. Luo, T. Wu, G. Y. Wang, and X. H. Chen, Transport properties of single-crystalline $\mathrm{Cu}_{x} \mathrm{TiSe}_{2}$ $(0.015 \leqslant x \leqslant 0.110)$, Phys. Rev. B 76, 024513 (2007).

[14] S. L. Bud'ko, P. C. Canfield, E. Morosan, R. J. Cava, and G. M. Schmiedeshoff, Thermal expansion and effect of pressure on superconductivity in $\mathrm{Cu}_{x} \mathrm{TiSe}_{2}$, J. Phys. Condens. Matter 19, 176230 (2007).

[15] B. Hildebrand, T. Jaouen, C. Didiot, E. Razzoli, G. Monney, M.-L. Mottas, A. Ubaldini, H. Berger, C. Barreteau, H. Beck, D. R. Bowler, and P. Aebi, Short-range phase coherence and origin of the $1 T$-TiSe ${ }_{2}$ charge density wave, Phys. Rev. B 93, 125140 (2016).

[16] S. Y. Li, G. Wu, X. H. Chen, and L. Tailefer, Single-Gap s-Wave Superconductivity near the Charge-Density-Wave Quantum Critical Point in $\mathrm{Cu}_{x} \mathrm{TiSe}_{2}$, Phys. Rev. Lett. 99, 107001 (2007).

[17] E. Morosan, L. Li, N. P. Ong, and R. J. Cava, Anisotropic properties of the layered superconductor $\mathrm{Cu}_{0.07} \mathrm{TiSe}_{2}$, Phys. Rev. B 75, 104505 (2007).

[18] A. F. Kusmartseva, B. Sipos, H. Berger, L. Forró, and E. Tutiš, Pressure Induced Superconductivity in Pristine $1 T-\mathrm{TiSe}_{2}$, Phys. Rev. Lett. 103, 236401 (2009).

[19] Y. I. Joe, X. M. Chen, P. Ghaemi, K. D. Finkelstein, G. A. de la Peña, Y. Gan, J. C. T. Lee, S. Yuan, J. Geck, G. J. MacDougall, T. C. Chiang, S. L. Cooper, E. Fradkin, and P. Abbamonte, Emergence of charge density wave domain walls above the superconducting dome in $1 T-\mathrm{TiSe}_{2}$, Nat. Phys. 10, 421 (2014).

[20] H. R. Friend, D. Jérome, and A. D. Yoffe, High-pressure transport properties of $\mathrm{TiS}_{2}$ and $\mathrm{TiSe}_{2}$, J. Phys. C Solid State Phys. 15, 2183 (1982).

[21] C. S. Snow, J. F. Karpus, S. L. Cooper, T. E. Kidd, and T.-C. Chiang, Quantum Melting of the Charge-Density-Wave State in $1 T$-TiSe 2 , Phys. Rev. Lett. 91, 136402 (2003).

[22] K. Sugawara, Y. Nakata, R. Shimizu, P. Han, T. Hitosugi, T. Sato, and T. Takahashi, Unconventional charge-densitywave transition in monolayer $1 T$-TiSe 2 , ACS Nano 10, 1341 (2015).

[23] P. Chen, Y.-H. Chan, X.-Y. Fang, Y. Zhang, M. Y. Chou, S.K. Mo, Z. Hussain, A.-V. Fedorov, and T.-C. Chiang, Charge density wave transition in single-layer titanium diselenide, Nat. Commun. 6, 8943 (2015).

[24] L. J. Li, E. C. T. O’Farrell, K. P. Loh, G. Eda, B. Özyilmaz, and A. H. Castro Neto, Controlling many-body states by the electric-field effect in a two-dimensional material, Nature 529, 185 (2016).

[25] L. J. Li, W. J. Zhao, B. Liu, T. H. Ren, G. Eda, and K. P. Loh, Enhancing charge-density-wave order in $1 T$-TiSe ${ }_{2}$ nanosheet by encapsulation with hexagonal boron nitride, Appl. Phys. Lett. 109, 141902 (2016).

[26] M. Maschek, S. Rosenkranz, R. Hott, R. Heid, M. Merz, D. A. Zocco, A. H. Said, A. Alatas, G. Karapetrov, S. Zhu, J. van Wezel, and F. Weber, Superconductivity and hybrid soft modes in $\mathrm{TiSe}_{2}$, Phys. Rev. B 94, 214507 (2016).

[27] D. Venkateshwarlu, S. S. Samatham, M. Gangrade, and V. Ganesan, Superconductivity in $\mathrm{Cu}_{0.08} \mathrm{TiSe}_{2}$, in Proceedings of the 56th DAE Solid State Physics Symposium 2011, edited by R. Mittal, A. K. Chauhan, and R. Mukhopadhyay, AIP Conf. Proc. No. 1447 (AIP, New York, 2012), p. 887.

[28] A. Ubaldini, J. Jacimovic, N. Ubrig, and E. Giannini, Chloridedriven chemical vapor transport method for crystal growth of transition metal dichalcogenides, Cryst. Growth Des. 13, 4453 (2013).

[29] I. R. Walker, Nonmagnetic piston-cylinder pressure cell for use at $35 \mathrm{kbar}$ and above, Rev. Sci. Instrum. 70, 3402 (1999).

[30] T. C. Kobayashi, H. Hidaka, H. Kotegawa, K. Fujiwara, and M. I. Eremets, Nonmagnetic indenter-type high-pressure cell for magnetic measurements, Rev. Sci. Instrum. 78, 023909 (2007).

[31] A. Eiling and J. S. Schilling, Pressure and temperature dependence of electrical resistivity of $\mathrm{Pb}$ and $\mathrm{Sn}$ from $1-300 \mathrm{~K}$ and 0-10 GPa-use as continuous resistive pressure monitor accurate over wide temperature range; superconductivity under pressure in Pb, Sn and In, J. Phys. F Metal Phys. 11, 623 (1981).

[32] S. Lee, J. Jang, S.-I. Kim, S.-G. Jung, J. Kim, S. Cho, S. W. Kim, J. Y. Rhee, K.-S. Park, and T. Park, Origin of extremely large magnetoresistance in the candidate type-II Weyl semimetal $\mathrm{MoTe}_{2-x}$, Sci. Rep. 8, 13937 (2018).

[33] X.-C Pan, X. Chen, H. Liu, Y. Feng, Z. Wei, Y. Zhou, Z. Chi, L. Pi, F. Yen, F. Song, X. Wan, Z. Yang, B. Wang, G. Wang, and Y. Zhang, Pressure-driven dome-shaped superconductivity and electronic structural evolution in tungsten ditelluride, Nat. Comm. 6, 7805 (2015).

[34] T. Park, H. Lee, I. Martin, X. Lu, V. A. Sidorov, K. Gofryk, F. Ronning, E. D. Bauer, and J. D. Thompson, Textured Superconducting Phase in the Heavy Fermion CeRhIn ${ }_{5}$, Phys. Rev. Lett. 108, 077003 (2012).

[35] N. R. Werthamer, E. Helfand, and P. C. Hohenberg, Temperature and purity dependence of the superconducting critical field, $H_{c 2}$. III. Electron spin and spin-orbit effects, Phys. Rev. 147, 295 (1966).

[36] S. T. Ruggiero, T. W. Barbee, Jr., and M. R. Beasley, Superconductivity in Quasi-Two-Dimensional Layered Composites, Phys. Rev. Lett. 45, 1299 (1980).

[37] A. Nader and A. LeBlanc, Resistivity anisotropy and charge density wave in $1 T$-TiSe 2 , Indian J. Phys. 87, 363 (2013).

[38] S. Seo, E. Park, E. D. Bauer, F. Ronning, J. N. Kim, J.-H. Shim, J. D. Thompson, and T. Park, Controlling superconductivity by tunable quantum critical points, Nat. Comms. 6, 6433 (2015). 